\title{
AVALIAÇÃO DA RESOLUTIVIDADE E EFETIVIDADE DA ATENÇÃO PRIMÁRIA À SAÚDE: REVISÃO INTEGRATIVA DE LITERATURA
}

EVALUATING PROBLEM-SOLVING AND EFFECTIVENESS OF PRIMARY HEALTH CARE: INTEGRATIVE LITERATURE REVIEW

EVALUACIÓN DE LA RESOLUCIÓN DE PROBLEMAS Y LA EFECTIVIDAD DE LA ATENCIÓN PRIMARIA DE SALUD: REVISIÓN INTEGRADORA DE LITERATURA

\author{
Maristela Inês Osawa Vasconcelos 1 \\ Aparecida Lara Carlos Xavier ${ }^{2}$ \\ Maksoane Nobre do Nascimento ${ }^{3}$ \\ Yanka Alcântara Cavalcante ${ }^{4}$ \\ Sibele Pontes Rocha ${ }^{5}$ \\ Josiane da Silva Gomes 6
}

Palavras-chave: Efetividade; Atenção Primária à Saúde; Avaliação em Saúde; Resolutividade.

Keywords: Effectiveness; Primary Health Care; Health Evaluation; Problem-Solving.

Palabras clave: Efectividad; Atención Primaria de Salud; Evaluación en Salud; Resolución de Problemas.

Submetido: $17 / 03 / 2018$

Aprovado: 26/04/2018

\section{Autor(a) para Correspondência:} Josiane da Silva Gomes End: Rua Humberto Lopes, 258, Ap.A, Domingos Olímpio

Sobral-Ceará. CEP: 62.022-304 E-mail: josianegomes4@hotmail.com

\section{RESUMO}

Este artigo analisa métodos de avaliação da efetividade e resolutividade na atenção primária à saúde (APS). Trata-se de revisão integrativa de literatura considerando o período de 2008 a 2017, com buscas na Biblioteca Virtual em Saúde (BVS) e na Scientific Electronic Library Online (SCIELO) e uso dos descritores em ciências da saúde (DeCS) "efetividade", "atenção primária à saúde" e "avaliação em saúde", além do termo-chave "resolutividade". Por meio da aplicação dos critérios de inclusão e exclusão, 13 publicações foram selecionadas para compor a amostra do estudo, analisadas a partir das evidências científicas. Ao analisar essas publicações, identificou-se que a efetividade e resolutividade na APS estão interligadas à avaliação positiva por parte dos usuários acerca das ações realizadas pelos serviços de saúde. Constatou-se que uma assistência aos usuários de boa qualidade contribui com a boa gestão. Identificar os processos que necessitam de redirecionamento de gastos junto aos setores administrativo e financeiro do sistema de saúde possibilita a obtenção de resultados satisfatórios dos serviços de saúde.

1. Professora no Curso de Graduação em Enfermagem da Universidade Estadual Vale do Acaraú (UVA) e no Mestrado Acadêmico em Saúde da Família da Universidade Federal do Ceará (UFC). E-mail: maristela.osawa@ gmail.com

2. Aluna de Graduação em Enfermagem na UVA. Bolsista do Programa de Bolsas de Produtividade em Pesquisa. E-mail: laraxavier5@gmail.com

3. Aluna de Graduação em Enfermagem na UVA. Bolsista do Programa de Bolsas de Produtividade em Pesquisa. E-mail: maksoane@gmail.com

4. Aluna de Graduação em Enfermagem na UVA. Bolsista do Programa de Bolsas de Produtividade em Pesquisa.

E-mail: yanka-AC2010@hotmail.com

5. Aluna do Mestrado Acadêmico em Saúde da Família da UFC. E-mail: sibelepontes63@gmail.com

6. Aluna do Mestrado Acadêmico em Saúde da Família da UFC. E-mail: josianegomes4@hotmail.com 


\section{ABSTRACT}

This article analyzes methods for evaluating effectiveness and problem-solving of primary health care (PHC). This is an integrative literature review considering the period from 2008 to 2017, with search in the Virtual Health Library (VHL) and the Scientific Electronic Library Online (SciELO) and use of the health sciences descriptors (DeCS) 'effectiveness,' 'primary health care,' and 'health evaluation,' in addition to the key term 'problem-solving.' By applying the inclusion and exclusion criteria, 13 publications were selected to be in the study sample, analyzed through scientific evidence. When analyzing these publications, we identified that effectiveness and problemsolving in the PHC are intertwined with positive assessment by users about the actions taken by the health services. We found out that good-quality care for users contributes to good management. Identifying the processes that need to redirect spending along with the administrative and financial sectors of the health system makes it possible to obtain satisfactory outcomes from health services.

\section{RESUMEN}

Este artículo analiza los métodos de evaluación de la efectividad y la resolución de problemas en la atención primaria de salud (APS). Esta es una revisión integradora de literatura considerando el período de 2008 a 2017 , con búsqueda en la Biblioteca Virtual en Salud (BVS) y la Scientific Electronic Library Online (SCiELO) y uso de los descriptores en ciencias de la salud (DeCS) "efectividad", "atención primaria de salud" y "evaluación en salud", además del término clave "resolución de problemas". Mediante la aplicación de los criterios de inclusión y exclusión, se seleccionaron 13 publicaciones para componer la muestra del estudio, analizadas a través de las evidencias científicas. Al analizar estas publicaciones, se identificó que la efectividad y resolución en la APS están interconectadas a la evaluación positiva por parte de los usuarios sobre las acciones realizadas por los servicios de salud. Se constató que una asistencia a los usuarios de buena calidad contribuye a la buena gestión. Identificar los procesos que necesitan redirigir el gasto junto con los sectores administrativo y financiero del sistema de salud permite obtener resultados satisfactorios de los servicios de salud.

\section{INTRODUÇÃO}

A atenção primária à saúde (APS) representa 0 primeiro nível de acesso dos usuários ao sistema de saúde e desenvolve ações de prevenção, promoção, proteção e reabilitação da saúde, visando ao atendimento das demandas de saúde dos indivíduos, das famílias e das comunidades. A APS é considerada uma estratégia fundamental para aumentar a efetividade e resolutividade dos serviços, constituindo referência no cenário mundial para reduzir as iniquidades existentes no setor saúde ${ }^{1}$.

É importante esclarecer que a efetividade é o grau em que determinada intervenção ou tecnologia traz benefícios aos indivíduos de uma população; na APS, a efetividade se refere à atenção capaz de prevenir doenças ou controlar episódios agudos decorrentes de condições crônicas ${ }^{2}$. Por sua vez, a resolutividade é a capacidade de solucionar problemas de saúde de indivíduos em situação de vulnerabilidade social e biológica, norteada pelos seguintes atributos: a) acesso; b) longitudinalidade; c) integralidade; e d) coordenação ${ }^{3}$.

As pesquisas de avaliação em saúde que monitoram a qualidade e capacidade resolutiva dos serviços ofertados pela APS no Brasil alcançaram maior destaque a partir de iniciativas federais em 2004, com foco especial na Estratégia Saúde da Família $(E S F)^{4}$ - considerada a porta de entrada do usuário no Sistema Único de Saúde (SUS). A avaliação nesse nível de atenção se mostra relevante para identificar as fragilidades que dificultam sua organização e operacionalização $0^{5}$.

Desse modo, a avaliação em saúde tem configurado um importante instrumento para a avaliação da qualidade dos serviços da APS, possibilitando discussões sobre sua capacidade resolutiva e sua efetividade, além de fornecer respostas sobre as políticas e os programas, para abrir espaço a reestruturações alinhadas às reais necessidades dos indivíduos e da coletividade ${ }^{6}$.

Diante do exposto, compreende-se que houve muitos avanços na APS, principalmente devido às políticas e aos programas criados com o intuito de garantir o direito à saúde dos diferentes grupos populacionais atendidos pela ESF. No entanto, questiona-se se tais projetos atingem seus objetivos, pois se observa que esse nível de atenção tem vivenciado problemas relativos à estrutura física dos serviços, à escassez de recursos e à insuficiência de profissionais, o que reforça as dúvidas quanto à efetividade da APS para atender às demandas de 
saúde da população ${ }^{7}$.

Este estudo se justifica pela necessidade de compreender os processos implantados na APS, com vistas à garantia de sua efetividade e resolutividade, a partir da identificação das estratégias de avaliação das ações desenvolvidas pela ESF. Nesse sentido, o estudo levantou na literatura científica produções relacionadas aos métodos de avaliação da efetividade e resolutividade na APS.

\section{METODOLOGIA}

Este estudo se caracteriza como uma revisão integrativa - método que reúne, analisa e sintetiza a produção científica sobre uma temática específica, a fim de obter uma compreensão completa do fenômeno que se almeja estudar ${ }^{8}$. A construção de uma revisão integrativa contempla as seguintes fases: a) identificação do tema e elaboração da pergunta norteadora; b) busca ou amostragem na literatura (definindo os critérios de inclusão e exclusão); c) categorização dos estudos; d) análise crítica dos estudos incluídos; e) interpretação dos resultados e síntese do conhecimento (apresentação da revisão) ${ }^{9}$.

Para seguir o padrão metodológico proposto, buscou-se responder a seguinte pergunta norteadora:

- Quais métodos vêm sendo utilizados para a avaliação da efetividade e resolutividade na APS?

As buscas, considerando o período de 2008 a 2017, foram realizadas em junho e julho de 2017 na Biblioteca Virtual em Saúde (BVS) e na Scientific Electronic Library Online (SciELO), com uso dos descritores em ciências da saúde (DeCS) "efetividade", "atenção primária à saúde" e "avaliação em saúde", além do termo-chave "resolutividade".

Recorremos a 5 cruzamentos entre os descritores e a palavra-chave em questão, mediados pelo operador booleano "and": a) "atenção primária à saúde" and "resolutividade"; b) "atenção primária à saúde" and "efetividade"; c) "atenção primária à saúde" and "resolutividade" and "efetividade"; d) "efetividade" and "atenção primária à saúde" and "avaliação em saúde"; e e) "resolutividade" and "atenção primária à saúde" and "avaliação em saúde".

Os critérios de inclusão foram: a) artigos nacionais disponiveis em texto integral; b) artigos em língua portuguesa; c) artigos publicados entre 2008 a 2017; e d) artigos que discutem as formas de avaliação da efetividade e resolutividade na APS.

Já os critérios de exclusão foram: a) artigos não disponiveis em texto integral; b) artigos duplicados

\section{Quais métodos vêm sendo utilizados para a avaliação da efetividade $e$ resolutividade na APS?}

nas bases BVS e SciELO; e c) artigos que não abordam a temática proposta.

A coleta de dados foi mediada por um instrumento validado e adaptado pelas autoras, para sistematizar e organizar os estudos, minimizando o risco de erros ou omissão de informações ${ }^{10}$. 0 s estudos foram selecionados por: a) título; b) autor(es); c) ano de publicação; d) métodos; e e) nível de evidência científica.

Há 6 níveis de evidência científica:

- Nivel 1: evidências resultantes da metanálise de múltiplos estudos clínicos controlados e randomizados;

- Nivel 2: evidências obtidas em estudos individuais com delineamento experimental;

- Nivel 3: evidências de estudos quase experimentais;

- Nivel 4: evidências de estudos descritivos (não experimentais) ou com abordagem qualitativa;

- Nivel 5: evidências provenientes de relatos de caso ou relatos de experiência; e

- Nível 6: evidências baseadas em opiniões de especialistas ${ }^{8}$.

As publicações foram selecionadas com base na leitura de títulos e resumos, considerando os critérios de inclusão e exclusão. Os artigos pré-selecionados foram lidos na íntegra e analisados segundo os níveis de evidência, sendo sistematizados para estabelecer a amostra da revisão. Os artigos selecionados foram sintetizados e discutidos a partir da literatura pertinente.

\section{RESULTADOS}

As buscas na BVS identificaram 269 publicações; a leitura dos títulos e resumos levou à exclusão de 240 publicações (221 não tinham relação com o tema e 19 estavam duplicadas). Após essa pré-seleção, restaram 29 artigos, dos quais foram excluídos 19 após a leitura integral (por não abordarem métodos de 
avaliação da resolutividade e efetividade especificamente). Restaram, portanto, 10 artigos para compor a amostra deste estudo.

As buscas na SciELO foram realizadas com os mesmos cruzamentos, resultando em 159 publicações; 136 publicações foram excluídas após a leitura dos títulos e resumos por não abordarem diretamente a temática investigada e 20 foram excluídas por estar duplicadas, restando 3 artigos.

A Figura 1 resume a amostra final desta revisão integrativa.

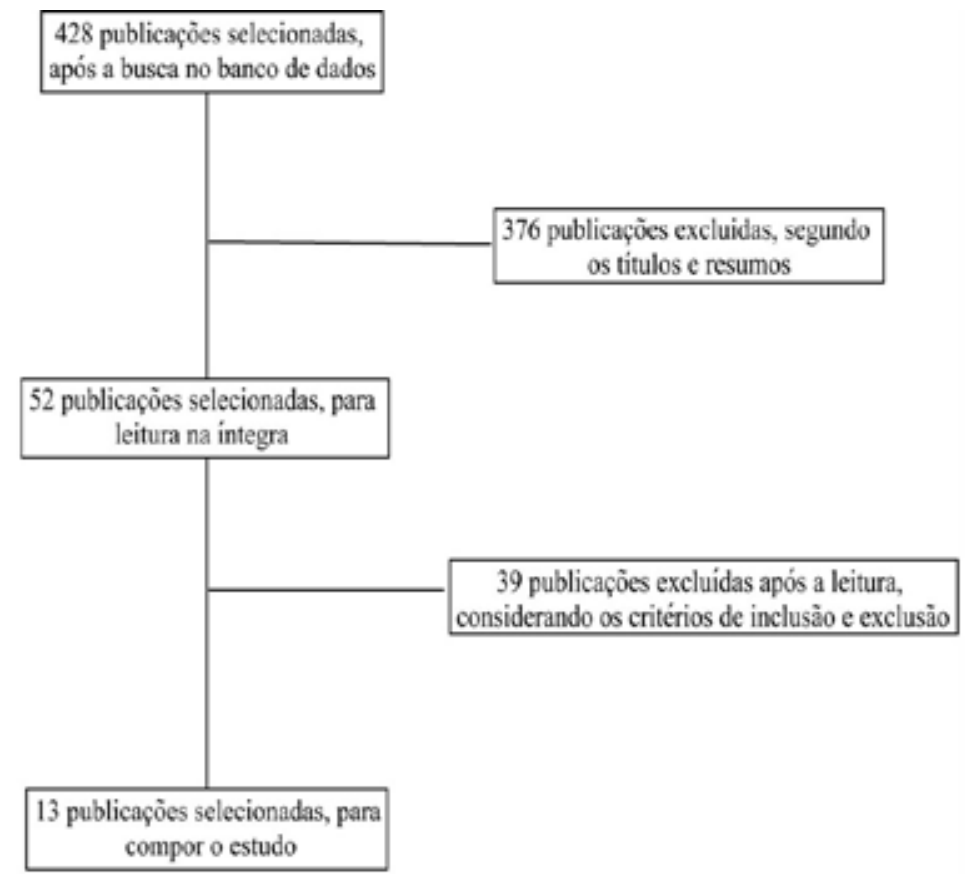

Figura 1. Distribuição dos artigos incluídos na amostra final da revisão integrativa. Fonte: Elaborada pelas autoras.

A Tabela 1 sistematiza os 13 estudos selecionados para a amostra final desta revisão integrativa.

Tabela 1 - Caracterização dos artigos incluídos na amostra final da revisão integrativa.

\begin{tabular}{llll}
\hline Titulo Autores Ano & Métodos Nivel de \\
evidência
\end{tabular}

1. Resolutividade dos serviços de saúde por inquérito domiciliar: percepção do usuário ${ }^{22}$
Turrini RNT, Lebrão $M L$, Cesar CLG
Tipo de estudo: descritivo.

Coleta: banco de dados do

2008 inquérito domiciliar, morbidade referida e utilização de serviços de saúde no ERSA-12.

2. Avaliação de efetividade da Atenção Básica à Saúde em municípios das regiões Sul e Nordeste do Brasil: contribuições metodológicas $^{16}$

3. Câncer cérvico-uterino como condição marcadora: uma proposta de avaliação da atenção básica ${ }^{17}$

Facchini LA, Piccini RX, Tomasi E, Thumé $E$, Teixeira VA, Silveira DS, et al.

Bottari CMS, Vasconcellos MM, Mendonça MHM
Tipo de estudo: transversal.

2008 Coleta: grupos de comparação e avaliações em saúde pública.
4. Resolutividade da assistência e satisfação de usuários da Estratégia Saúde da Família ${ }^{19}$
Rosa RB, Pelegrini AHW, Lima MADS
Tipo de estudo: qualiquantitativo.

Coleta: ELB-PROESF em municípios com população acima de 100 mil habitantes no estado do Rio de Janeiro.

Tipo de estudo: quantiqualitativo.

2011

Coleta: formulário estruturado e entrevista semiestruturada. 
5. Regulação do acesso ao cuidado na atenção primária: percepção dos usuários ${ }^{18}$
Clares JWB, Silva LMS, Dourado HHM, Lima LL
2011

Campos RT0, Campos

GWS, Ferrer AL, Corrêa CRS, Madureira PR, Gama CAP, et al.
Tipo de estudo: exploratóriodescritivo, com abordagem qualitativa.

Coleta: entrevista

semiestruturada e análise de conteúdo.

Tipo de estudo: avaliativo, qualitativo, participativo, com

triangulação de métodos e referencial teórico da inovadoras na organização da atenção primária à saúde ${ }^{20}$

2012 hermenêutica.

Coleta: divisão de grupos para análise e comparação da aplicação de escalas nos 2 distritos de saúde mais populosos de Campinas (SP).

Tipo de estudo: revisão conceitual.
7. Avaliação de desempenho de sistemas de saúde: um modelo de análise ${ }^{2}$
Viacava F, Ugá MAD,

Porto S, Laguardia J, Moreira RS
2012

Coleta: indicadores sugeridos

na segunda etapa do PROADESS

e sintese dos resultados obtidos.
8. Acessibilidade aos serviços de saúde: uma análise a partir da atenção básica em Pernambuco ${ }^{23}$
Albuquerque MSV, Lyra TM, Farias SF, Mendes MFM, Martelli PJL
Tipo de estudo: quantitativo 2014 descritivo, de corte transversal. 4 Coleta: dados do PMAQ-AB.
9. 0 acesso e a dificuldade na resolutividade do cuidado da criança na atenção primária à saúde ${ }^{21}$

10. A resolutividade em saúde bucal na atenção básica como instrumento para avaliação dos modelos de $\operatorname{atenção~}{ }^{3}$
Finkler AL, Viera CS, Tacla MTGM, Toso BRGO
Tipo de estudo: qualitativo.

Coleta: hermenêutica e observação não participante.
Tipo de estudo: ecológico, retrospectivo.

Coleta: indicadores de acesso Cortellazzi KL, Ambrosano GMB, Meneghim MC, Faria $E T$, Mialhe FL, et al.
2014

ao tratamento odontológico e percentual de cobertura populacional em tratamento completado inicial (TCI).
11. Avaliação da longitudinalidade em unidades de atenção primária à saúde $\mathrm{e}^{12}$
Frank BRB, Viera CS, Ross C, Obregón PL, Toso BRG0

Tipo de estudo: quantitativo.

Coleta: Primary Care Assessment

Tool (PCATool), versão criança, validado no Brasil em 2010.

Tipo de estudo: avaliativo, transversal, com abordagem quantitativa.

12. Avaliação do desempenho da atenção básica nos municípios brasileiros com indicador

Miclos PV, Calvo MCM, Colussi CF

2015

Coleta: indicador sintético desenvolvido pelo Núcleo de

Extensão e Pesquisa em Avaliação em Saúde da Universidade Federal de Santa Catarina (Nepas/UFSC).

Tipo de estudo: avaliativo descritivo.

13. Estratégias para consolidação da coordenação do cuidado pela atenção básica ${ }^{4}$
Almeida PF, Marin J, Casotti E
2017

Coleta: questionários aplicados

a 1.313 usuários e 324 equipes de atenção básica no município do Rio de Janeiro.

Fonte: Elaborada pelas autoras. 
A partir da análise dessa amostra bibliográfica, observou-se que algumas profissões do setor saúde estavam mais presentes nas discussões sobre as avaliações da resolutividade e efetividade na APS, dentre elas: Enfermagem (artigos n. 1, 3-5, 9 e 10); Medicina (artigos n. 2, 6 e 7); 0dontologia (artigos n. 11 e 12); Serviço Social (artigo n. 8); e Psicologia (artigo n. 13).

Quanto ao grau de evidência, a Tabela 1 indica $61,53 \%$ de estudos com nível 4 de evidência (artigos n. 4, 5, 7-10, 12 e 13), divididos entre estudos descritivos e estudos com abordagem qualitativa; $30,76 \%$ de estudos com nível 3 (artigos n. 1, 3, 6 e 11), onde se situam os estudos quase experimentais; e $7,6 \%$ de estudos com nível 2 (artigo n. 1) - de delineamento experimental.

No que tange ao recorte temporal das publicações, foram identificados 3 publicações de 2008 (artigos $n$. 1-3), 3 publicações de 2011 (artigos n. 4-6), 1 publicação de 2012 (artigo n. 7), 3 publicações de 2014 (artigos n. 8-10), 2 publicações de 2015 (artigos n. 11 e 12) e 1 publicação de 2017 (artigo n. 13).

No que se refere ao delineamento metodológico dos artigos analisados, 8 estudos eram quantitativos, 6 estudos eram qualitativos, 5 apresentavam abordagem descritiva, 4 tinham abordagem avaliativa, 2 estudos eram exploratórios, 1 estudo era transversal e havia 1 revisão conceitual e 1 estudo ecológico retrospectivo.

\section{DISCUSSÃO}

Constatou-se a relevância do tema em relação ao cenário nacional e às modificações das práticas em saúde, pois tais estudos contribuem para 0 fortalecimento do SUS na busca por soluções para ampliar a efetividade e resolutividade dos serviços ofertados pela APS ${ }^{11}$.

0s resultados demonstram que a APS requer compromisso político para responder de modo efetivo e resolutivo às demandas de saúde dos indivíduos e as práticas assistenciais devem ser de boa qualidade e inovadoras ${ }^{12}$. Entretanto, a APS enfrenta vários desafios, como: a) oferta de serviços e infraestrutura inadequadas; e b) desarticulação entre acolhimento dos usuários e coordenação da atenção. Isso interfere no processo de trabalho capaz de satisfazer as reais necessidades dos usuários ${ }^{13}$.

Dentre os estudos que integram a revisão, 6 publicações provêm da enfermagem - área de vital importância para as atribuições da APS com grande

\section{... a APS requer compromisso político para responder de modo efetivo $e$ resolutivo às demandas de saúde...}

repercussão na realidade desse nível de atenção em âmbito nacional ${ }^{14}$.

Notou-se um aumento do número de publicações que abordam a avaliação dos serviços de saúde com foco na APS em 2014. Sugere-se que tal aumento se deve à criação do Programa de Melhoria do Acesso e da Qualidade da Atenção Básica (PMAQ-AB), em 2011, que possibilita avaliar os serviços de saúde segundo a Política Nacional da Atenção Básica (PNAB), criada em $2006^{15}$.

Outro aspecto analisado se refere ao nível de evidência científica - a maioria dos artigos foi considerada de média qualidade, pois seu delineamento metodológico se pautou em estudos descritivos (não experimentais) ou em abordagens qualitativas, o que os levou a não apresentar um alinhamento que permita sua aplicação em diferentes realidades e contextos; entretanto, proporcionam informações importantes acerca da efetividade e resolutividade da APS.

Vale ressaltar que os estudos selecionados trazem diversas abordagens metodológicas para avaliação dos resultados no setor saúde, desde a utilização de escalas, indicadores e instrumentos, em sua maioria criados e validados pelos próprios autores; todavia, esses estudos têm em comum os resultados obtidos por meio do PMAQ-AB.

Ao analisar o conteúdo da amostra foram identificadas 9 estratégias metodológicas.

Algumas pesquisas apresentam como semelhança o chamado estudo de linha de base (ELB), que avalia o desempenho da APS na implementação do Projeto de Expansão e Consolidação do Saúde da Família (PROESF), utilizando dados secundários do DataSUS. As questões dos formulários englobam gestão, controle social, estrutura, profissionais e usuários dos serviços de saúde ${ }^{16,17}$.

Outras publicações desenvolveram questionários com aplicação de entrevistas semiestruturadas, com o objetivo de avaliar a efetividade e resolutividade das 
ações inerentes à APS com base no discurso da população atendida nos serviços de saúde ${ }^{18,19}$. Também se identificou nessas pesquisas a elaboração de escalas e diários de campo e o uso dos métodos de análise temática e triangulação hermenêutica - com abordagem sócio-histórica para interpretação/ reinterpretação dos dados ${ }^{20,21}$.

observou-se que a maioria dos estudos com aplicação de questionários e formulários avaliou as variáveis direcionadas efetividade e resolutividade dos serviços por meio dos módulos I, II e III do $P M A Q-A B^{4,22,23}$. Alguns pesquisadores apresentam métodos diferenciados para análise da resolutividade, com uso de registros do escritório regional de saúde combinados ao inquérito domiciliar de morbidade referida, englobando 14 variáveis inerentes ao objeto de estudo 22 .

Um estudo do tipo revisão conceitual, realizado a partir da proposta formulada pelo Projeto de Avaliação de Desempenho de Sistemas de Saúde (PROADESS), elencou subdimensões de avaliação do desempenho dos serviços de saúde: a) efetividade; b) acesso; c) eficiência; e d) adequação. No que se refere à efetividade, esse estudo reuniu os indicadores em 3 grupos: a) mortes evitáveis; b) morbidade evitável; e c) internações evitáveis - cada um desses indicadores tem seu subgrupo de taxas epidemiológicas, que foram utilizadas para mensurar a efetividade da APS 2 .

Outro estudo adotou abordagem distinta, com uso da base de dados do Sistema de Informação em Saúde para a Atenção Básica (SISAB) e do relatório de avaliação de saúde bucal, para analisar a resolutividade dos serviços de tratamento bucal ${ }^{21}$.

E outra publicação recorreu a um instrumento de coleta de dados internacional, adaptado e validado para o Brasil, denominado PrimaryCare Assessment Tool (PCATool-Brasil); por meio dele foram investigadas as variáveis de um atributo quanto à distribuição de suas frequências absolutas e relativas e de suas médias e medianas.

Nesta revisão, pôde-se verificar que, apesar dos desafios para atingir bons níveis de efetividade e resolutividade na APS, os avanços se sobrepõem às dificuldades, resultando em maior número de ações integradas na APS, por meio da implantação de programas como a Política Nacional de Atenção Integral à Saúde da Mulher (PNAISM) e a Política Nacional de Saúde Bucal (PNSB) 24,25.

A integração de ações na APS pode ser mencionada a partir de experiências relatadas em estudos realizados nos estados de São Paulo e do Rio de

\section{...os avanços se \\ sobrepõem às \\ dificuldades, \\ resultando em maior \\ número de ações integradas na APS...}

Janeiro - a introdução dessas políticas na APS contribui para a expansão da cobertura da ESF e, consequentemente, para a garantia do direito à saúde do usuário ${ }^{3,17}$.

Nos estudos incluídos nesta revisão, observou-se que a ideia de efetividade na APS se relaciona aos resultados das ações em saúde, sendo imprescindível que estas tragam benefícios para o indivíduo de determinada população e mostrem-se adequadas para avaliar o cumprimento de objetivos, metas e funções de ações programáticas na ESF2,16. A compreensão da resolutividade na APS mediada pela literatura envolve 4 fatores: a) oferta de serviços; b) infraestrutura; c) acolhimento; e d) coordenação da atenção. Nesse sentido, é imprescindivel que os serviços correspondam às demandas dos usuários ${ }^{19,21,22,26}$ e os estudos indicam que a APS efetiva e resolutiva está interligada à avaliação positiva de ações e serviços de saúde por parte dos usuários.

Os estudos selecionados apontaram as possiveis medidas a ser implementadas para garantir a resolutividade e efetividade na APS, sendo fundamental ampliar os recursos profissionais, assistenciais e tecnológicos e a infraestrutura dos serviços ${ }^{11}$. Apesar das diferenças locais dos estados brasileiros, eles seguem as mesmas diretrizes e a mesma legislação para a estruturação da APS; contudo, as ações definidas pelos gestores diferem de um lugar para o outro, constituindo distintas formas de organização, que, por sua vez, produzem resultados distintos no setor saúde ${ }^{26}$.

Assim, os resultados dos estudos incluídos na amostra desta revisão podem proporcionar apoio a outras localidades, levando em consideração as peculiaridades dos sistemas locais para avaliar os modelos de atenção operantes $3,19,20,26$. As metodologias de avaliação adotadas pelos estudos em questão apresentam impacto significativo na saúde da comunidade ao identificar a melhoria da qualidade dos serviços ofertados pela APS, assim como seus problemas e suas falhas, levando à necessidade de 
buscar estratégias para correção e novos planejamentos ${ }^{27}$.

\section{CONCLUSÃO}

Este estudo constatou que, para a avaliação da efetividade e resolutividade na APS, mostra-se necessária a adoção de um processo criterioso e organizado - com metodologias claras e bem definidas -, que possibilite o levantamento da real situação de saúde em análise, pois o uso de diferentes métodos pode levar ao distanciamento dos objetivos da avaliação.

Também se identificou que a qualidade da assistência aos usuários colabora com a gestão, ao identificar os processos que necessitam de redirecionamento de gastos junto ao setor administrativo e financeiro do setor saúde, contribuindo para um resultado satisfatório dos serviços de saúde.

Pode-se apontar como limitação deste estudo a adoção de diversas abordagens metodológicas para avaliação dos serviços de saúde, uma vez que os métodos apresentam restrições ao analisar as ações em saúde na APS, pois os dados apresentados não refletem a realidade dos serviços de saúde considerando as especificidades locais. Nesse sentido, faz-se necessária a realização de estudos que mensurem com maior precisão a efetividade e resolutividade, com uso de parâmetros nacionais e regionais que avaliem todas as diretrizes e todos os fundamentos da PNAB.

As autoras esperam que este artigo contribua com os esforços de outros pesquisadores interessados em analisar as diversas metodologias adotadas na avaliação da efetividade e resolutividade na APS. A literatura ainda se mostra escassa nesse área do conhecimento, tendo em vista o reduzido número de publicações identificadas e selecionadas para esta revisão integrativa.

\section{CONTRIBUIÇÃO DAS AUTORAS}

Maristela Inês 0 sawa Vasconcelos contribuiu com a concepção, realização da pesquisa, e redação e revisão crítica do manuscrito. Aparecida Lara Carlos Xavier contribuiu com a concepção e realização da pesquisa e a redação do manuscrito. Maksoane Nobre do Nascimento e Yanka Alcântara Cavalcante contribuíram com a estruturação e redação do manuscrito. Sibele Pontes Rocha e Josiane da Silva Gomes contribuíram com a redação do manuscrito.

\section{REFERÊNCIAS}

1. Carrer A, Toso BRG0, Guimarães ATB, Conterno JR, Minosso KC. Efetividade da Estratégia Saúde da Família em unidades com e sem Programa Mais Médicos em município no oeste do Paraná, Brasil. Ciênc Saúde Colet [serial on the internet]. 2016 [cited 2018 Apr 11];21(9):2849-60. Available from: http://www.scielo.br/pdf/csc/v21n9/14138123-csc-21-09-2849.pdf

2. Viacava F, Ugá MAD, Porto S, Laguardia J, Moreira RS. Avaliação de desempenho de sistemas de saúde: um modelo de análise. Ciênc Saúde Colet [serial on the internet]. 2012 [cited $2018 \mathrm{Apr} 9$ ];17(4):921-34. Available from: http:// www.scielo.br/pdf/csc/v17n4/v17n4a14.pdf

3. Bulgareli J, Cortellazzi KL, Ambrosano GMB, Meneghim MC, Faria ET, Mialhe FL, et al. A resolutividade em saúde bucal na atenção básica como instrumento para avaliação dos modelos de atenção. Ciênc Saúde Colet [serial on the internet]. 2014 [cited 2018 Apr 11];19(2):383-91. Available from: http://www.scielo.br/pdf/csc/v19n2/1413-8123csc-19-02-00383.pdf

4. Almeida PF, Marin J, Casotti E. Estratégias para consolidação da coordenação do cuidado pela atenção básica. Trab Educ Saúde [serial on the internet]. 2017 [cited 2018 Apr 9];15(2):373-98. Available from: http://www. scielo.br/pdf/tes/v15n2/1678-1007-tes-1981-7746sol00064.pdf

5. Miclos PV, Calvo MCM, Colussi CF. Avaliação do desempenho da atenção básica nos municípios brasileiros com indicador sintético. Saúde Debate [serial on the internet]. 2015 [cited 2018 Apr 11];39(107):984-96. Available from: http://www.scielo.br/pdf/sdeb/ v39n107/0103-1104-sdeb-39-107-00984.pdf

6. Sancho LG, Dain S. Avaliação em saúde e avaliação econômica em saúde: introdução ao debate sobre seus pontos de interseção. Ciênc Saúde Colet [serial on the internet]. 2012 [cited 2018 Apr 9];17(Suppl 3):765-74. Available from: http://www.scielo.br/pdf/csc/v17n3/ v17n3a24.pdf

7. Braga LM, Torres LM, Ferreira VM. Condições de trabalho e fazer em enfermagem. Revista de Enfermagem da UFJF [serial on the internet]. 2015 [cited 2018 Apr 10];1(1):5563. Available from: http://www.ufjf.br/ revistadeenfermagem/files/2015/05/10-Revista-deEnfermagem-C07.pdf

8. Souza MT, Silva MD, Carvalho R. Revisão integrativa: o que é e como fazer. Einstein [serial on the internet]. 2010 [cited 2017 Aug 8];8(1):102-6. Available from: http://www. scielo.br/pdf/eins/v8n1/pt 1679-4508-eins-8-1-0102.pdf

9. Mendes KDS, Silveira RCCP, Galvão CM. Revisão integrativa: método de pesquisa para a incorporação de evidências na saúde e na enfermagem. Texto \& Contexto Enferm [serial on the internet]. 2008 [cited 2017 Aug 8];17(4):758-64. Available from: http://www.scielo.br/pdf/ tce/v17n4/18.pdf 
10. Ursi ES. Prevenção de lesões de pele no perioperatório: revisão integrativa da literatura. Ribeirão Preto (SP): Universidade de São Paulo; 2005.

11. Rodrigues LBB, Silva PCS, Peruhype RC, Palha PF, Popolin $M P$, Crispim JA, et al. A atenção primária à saúde na coordenação das redes de atenção: uma revisão integrativa. Ciênc Saúde Colet [serial on the internet]. 2014 [cited 2018 Apr 9];19(2):343-52. Available from: http://www.scielo.br/ $\mathrm{pdf} / \mathrm{csc} / \mathrm{v} 19 \mathrm{n} 2 / 1413-8123-c s c-19-02-00343 . \mathrm{pdf}$

12. Frank BRB, Viera CS, Ross C, Obregón PL, Toso BRG0. Avaliação da longitudinalidade em unidades de atenção primária à saúde. Saúde Debate [serial on the internet]. 2015 [cited 2018 Apr 9];39(105):400-10. Available from: http://www.scielo.br/pdf/sdeb/v39n105/0103-1104sdeb-39-105-00400.pdf

13. Cubas MR, Faoro NT, Moysés ST, Carvalho DR. Avaliação da atenção primária à saúde: validação de instrumento para análise de desempenho dos serviços. Saúde Debate [serial on the internet]. 2017 [cited 2018 Apr 11];41(113): 47185. Available from: http://www.scielo.br/pdf/sdeb/ v41n113/0103-1104-sdeb-41-113-0471.pdf

14. Toso BRG0, Filippon J, Giovanella L. Atuação do enfermeiro na atenção primária no Serviço Nacional de Saúde da Inglaterra. Rev Bras Enferm [serial on the internet]. 2016 [cited 2018 Apr 11];69(1):182-91.Available from: http://www.scielo.br/pdf/reben/v69n1/0034-7167reben-69-01-0182.pdf

15. Brasil. Programa Nacional de Melhoria do Acesso e da Qualidade da Atenção Básica (PMAQ): manual instrutivo. Brasília (DF): Ministério da Saúde; 2012.

16. Facchini LA, Piccini RX, Tomasi E, Thumé E, Teixeira VA, Silveira DS, et al. Avaliação de efetividade da atenção básica à saúde em municípios das regiões Sul e Nordeste do Brasil: contribuições metodológicas. Cad Saúde Pública [serial on the internet]. 2008 [cited 2018 Apr 13];24(Suppl 1):159-72. Available from: http://www.scielo.br/pdf/csp/ v24s1/20.pdf

17. Bottari CMS, Vasconcellos MM, Mendonça MHM. Câncer cérvico-uterino como condição marcadora: uma proposta de avaliação da atenção básica. Cad Saúde Pública [serial on the internet]. 2008 [cited 2018 Apr 13];24(Suppl 1):S111-S122. Available from: https://www.scielosp.org/ $\mathrm{pdf} / \mathrm{csp} / \mathrm{v} 24 \mathrm{~s} 1 / 16 . \mathrm{pdf}$

18. Clares JWB, Silva LMS, Dourado HHM, Lima LL. Regulação do acesso ao cuidado na atenção primária: percepção dos usuários. Rev Enferm UERJ [serial on the internet]. 2011 [cited 2018 Apr 14];19(4):604-9. Available from: http:// www.facenf.uerj.br/v19n4/v19n4a17.pdf

19. Rosa RB, Pelegrini AHW, Lima MADS. Resolutividade da assistência e satisfação de usuários da Estratégia Saúde da Família. Rev Gaúch Enferm [serial on the internet]. 2011 [cited 2018 Apr 2014];32(2):345-51. Available from: http:// seer.ufrgs.br/index.php/RevistaGauchadeEnfermagem/ article/view/18574/12782
20. Campos RT0, Campos GWS, Ferrer AL, Corrêa CRS, Madureira PR, Gama CAP, et al. Avaliação de estratégias inovadoras na organização da atenção primária à saúde. Rev Saúde Pública [serial on the internet]. 2012 [cited $2018 \mathrm{Apr}$ 14];46(1):43-50. Available from: http://www.scielo.br/pdf/ rsp/v46n1/2502.pdf

21. Finkler AL, Viera CS, Tacla MTGM, Toso BRGO. 0 acesso e a dificuldade na resolutividade do cuidado da criança na atenção primária à saúde. Acta Paul Enferm [serial on the internet]. 2014 [cited $2018 \mathrm{Apr} 12$ ];27(6):548-53. Available from: http://www.scielo.br/pdf/ape/v27n6/1982-0194ape-027-006-0548.pdf

22. Turrini RNT, Lebrão ML, Cesar CLG. Resolutividade dos serviços de saúde por inquérito domiciliar: percepção do usuário. Cad Saúde Pública [serial on the internet]. 2008 [cited 2018 Apr 11];24(3):663-74. Available from: http:// www.scielo.br/pdf/csp/v24n3/20.pdf

23. Albuquerque MSV, Lyra TM, Farias SF, Mendes MFM, Martelli PJL. Acessibilidade aos serviços de saúde: uma análise a partir da atenção básica em Pernambuco. Saúde Debate [serial on the internet]. 2014 [cited 2018 Apr 12];38(Spec):182-94. Available from: http://www.scielo.br/ pdf/sdeb/v38nspe/0103-1104-sdeb-38-spe-0182.pdf

24. Ramos AL, Silva DP, Machado GMO, Oliveira EN, Lima DS. A atuação do enfermeiro da Estratégia Saúde da Família na prevenção do câncer de colo de útero. Sanare (Sobral, Online) [serial on the internet]. 2014 [cited $2018 \mathrm{Apr}$ 12];13(1):84-91. Available from: https://sanare.emnuvens. com.br/sanare/article/view/437/292

25. Carvalho TGS, Almeida AMB, Bezerra MIC. Percepção dos profissionais de saúde da atenção primária sobre educação permanente em saúde. Sanare (Sobral, Online) [serial on the internet]. 2016 [cited $2018 \mathrm{Apr} 12$ ];15(2):94-103. Available from: https://sanare.emnuvens.com.br/sanare/article/ view/1043/589

26. Miclos PV, Calvo MCM, Colussi CF. Avaliação do desempenho da atenção básica nos municípios brasileiros com indicador sintético. Saúde Debate [serial on the internet]. 2015 [cited 2018 Apr 13];39(107):984-96. Available from: http://www.scielo.br/pdf/sdeb/ v39n107/0103-1104-sdeb-39-107-00984.pdf

27. Ferreira VD, Oliveira JM, Maia MAC, Santos JS, Andrade RD, Machado GAB. Avaliação dos atributos da atenção primária à saúde em um município mineiro. Esc. Anna Nery Rev Enferm [serial on the internet]. 2016 [cited 2018 Apr 13];20(4):e20160104. Available from: http://www.scielo. br/pdf/ean/v20n4/en 1414-8145-ean-20-04-20160104.pdf
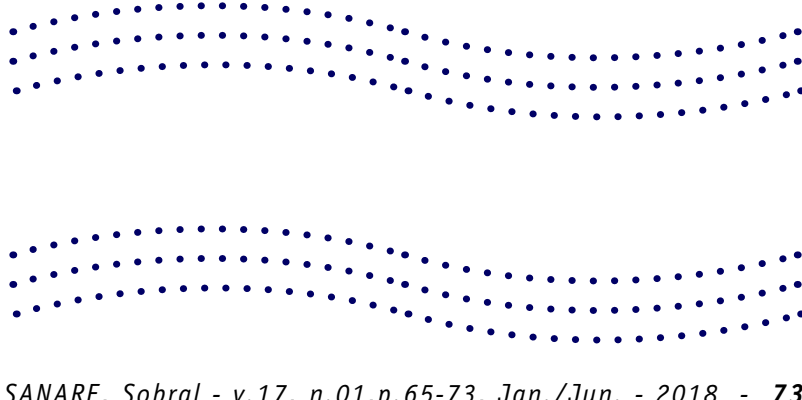